\title{
Stable kagome lattices from group IV elements
}

\author{
O. Leenaerts, ${ }^{1, *}$ B. Schoeters,${ }^{1,2, \dagger}$ and B. Partoens ${ }^{1, \ddagger}$ \\ ${ }^{1}$ Departement Fysica, Universiteit Antwerpen, Groenenborgerlaan 171, B-2020 Antwerpen, Belgium \\ ${ }^{2}$ IMEC, Kapeldreef 75, B-3001 Leuven, Belgium
}

(Received 14 November 2014; revised manuscript received 18 February 2015; published 10 March 2015)

\begin{abstract}
A thorough investigation of three-dimensional kagome lattices of group IV elements is performed with first-principles calculations. The investigated kagome lattices of silicon and germanium are found to be of similar stability as the recently proposed carbon kagome lattice. Carbon and silicon kagome lattices are both direct-gap semiconductors but they have qualitatively different electronic band structures. While direct optical transitions between the valence and conduction bands are allowed in the carbon case, no such transitions can be observed for silicon. The kagome lattice of germanium exhibits semimetallic behavior but can be transformed into a semiconductor after compression.
\end{abstract}

DOI: 10.1103/PhysRevB.91.115202

PACS number(s): 61.66.Bi, 61.50.-f, 71.15.Mb, 71.20.Mq

\section{INTRODUCTION}

In recent years, some new bulk phases of well-known elements such as carbon and silicon have been proposed. A remarkable example is silicite. This layered allotrope of silicon was reported [1] recently and can be formed by stacking dumbbell layers of silicene [3], which have been experimentally observed [2]. Each $\mathrm{Si}$ atom in this threedimensional (3D) structure has fourfold coordination, but the bond angles deviate considerably from the ideal $109.5^{\circ}$. Silicite has nonetheless an impressive cohesion of about $0.18 \mathrm{eV} /$ atom less than its cubic diamond counterpart [1].

Very recently, a new three-dimensional allotrope of carbon was predicted with some interesting properties [4]. This material, which was called a carbon kagome lattice (CKL), consists of $1 \mathrm{D}$ zigzag carbon chains locked together in a $2 \mathrm{D}$ kagome lattice structure [see Fig. 1(a)], resulting in a 3D material. Although this structure contains some $60^{\circ}$ bonds between $\mathrm{C}$ atoms, it was also found to be remarkably stable, comparable to $\mathrm{C}_{60}$ fullerene molecules. The presence of $\mathrm{C}$ triangles in the structure gives rise to orbital frustration, which causes a direct gap in the electronic band spectrum. Chen et al. [4] explicitly demonstrated the origin of this band gap by considering another related material, called an interpenetrated graphene network (IGN), which is pictured in Fig. 1(b). The IGN is semimetallic and it shows no orbital frustration. However, when the structure is compressed in one direction the orbital frustration increases and the IGN transforms into the CKL. This process is accompanied by the opening of a direct band gap in the electronic spectrum and is therefore strong evidence that the orbital frustration is indeed the origin of the gap. The unusual origin of the band gap makes the electronic spectrum of the carbon kagome lattice rather unique for a bulk elemental semiconductor. This is illustrated by its optical properties, which are similar to those of direct-gap semiconductors such as $\mathrm{GaN}$ and $\mathrm{ZnO}$ rather than other elemental semiconductors such as diamond and silicon [4].

The presence of frustration in kagome lattices is not unique to this case, but has a longer history in the condensed-matter literature. In fact, kagome lattices are well-known structures to study the physics of frustration [5-7]. An illustrative example is the spin frustration in kagome lattice structures that gives rise to the formation of spin ices, liquids, and glasses [8,9].

In this work we expand the study of carbon kagome lattices to the other group IV elements, silicon and germanium. Heavier elements are not considered here because we found them to be metallic. First we examine the dynamical stability of these structures through their phonon spectrum. The relative phase stability of the silicon and germanium kagome lattices ( $\mathrm{SiKL}$ and GeKL) is also investigated by comparing their cohesive energies to other elemental crystals such as 3D diamond and 2D graphenelike crystals. We perform a detailed examination of the electronic structure of SiKL and stress the differences with CKL. The character of the valence and conduction band states are determined and the possible optical transitions between these states are investigated. The electronic properties of germanium kagome lattices are also thoroughly studied. We find GeKL to be semimetallic and therefore investigate its behavior under strain.

\section{COMPUTATIONAL DETAILS}

We performed first-principles calculations within the density functional theory formalism as implemented in VASP $[10,11]$. The generalized gradient approximation of Perdew, Burke, and Ernzerhof (PBE-GGA) [12] was used for the exchange-correlation functional together with the more advanced Heyd-Scuseria-Ernzerhof hybrid functional (HSE06) [13] for band structure calculations. We made use of the projector augmented wave method [14-16] to reduce the size of the plane-wave basis set. The energy cutoff was set to $600 \mathrm{eV}, 400 \mathrm{eV}$, and $300 \mathrm{eV}$ for the cases of $\mathrm{C}, \mathrm{Si}$, and Ge systems, respectively. Integrations over the Brillouin zone were done with a $9 \times 9 \times 16$ Monkhorst-Pack $k$-point grid [17] for structural relaxations and band structure calculations, while a finer grid of $45 \times 45 \times 80$ was used for the calculation of the electronic density of states (DOS).

\section{RESULTS}

\footnotetext{
*ortwin.leenaerts@uantwerpen.be

†bob.schoeters@uantwerpen.be

†bart.partoens@uantwerpen.be
}

We start our study of the group IV kagome lattices by a detailed investigation of their structural properties. The crystal structure of the kagome lattice is shown in Fig. 1(a). The 

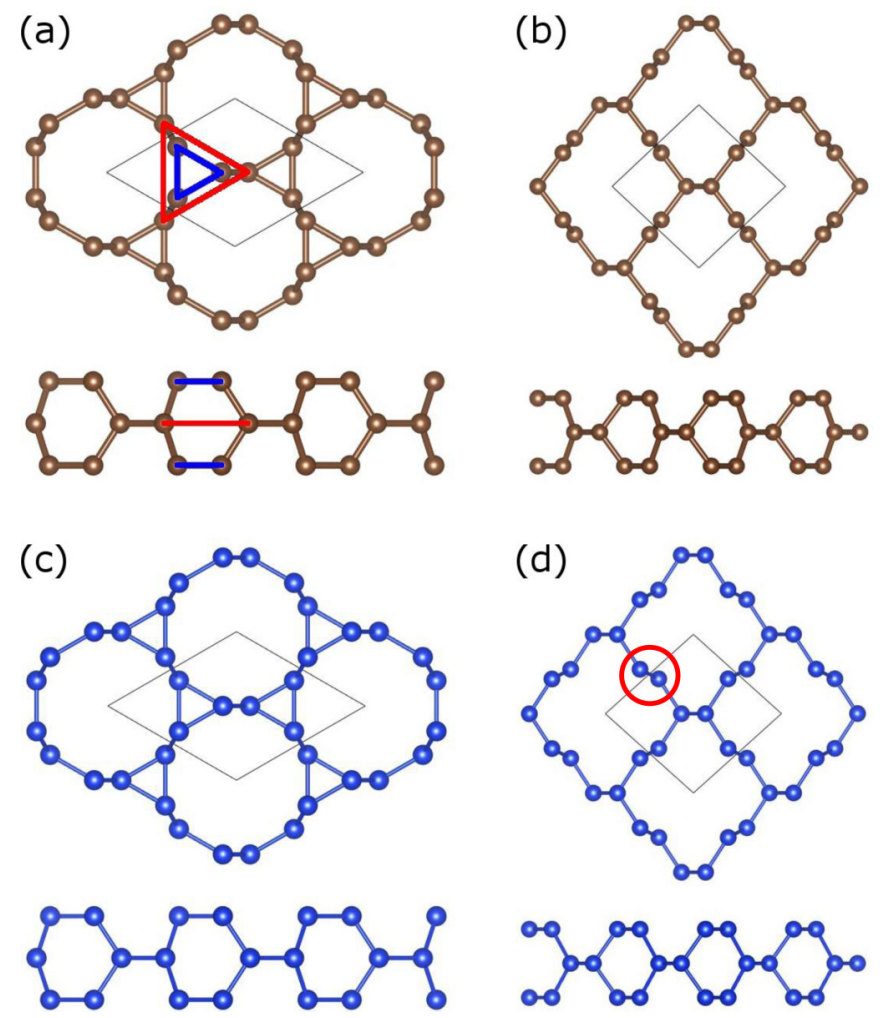

FIG. 1. (Color online) Top (top) and side (bottom) views of the crystal structure of (a) CKL, (b) IGN, (c) SiKL, and (d) ISiN. The unit cell is indicated with black lines.

kagome lattice consists of zigzag chains of atoms with their axes vertically aligned. These chains are bundled in groups of three in such a way that their atoms form alternatively small and larger triangles, as pictured in Fig. 1(a). Picturing every chain as a lattice point, the zigzag chains form a 2D triangular lattice in which every fourth lattice site is missing, i.e., a kagome lattice. Every atom in the structure has fourfold coordination, which makes this crystal structure well suited for group IV elements. We calculated the structure parameters of the first three group IV KLs with both the PBE-GGA and HSE06 functional and show the obtained results in Table I.

The PBE-GGA results for CKL are almost indistinguishable from those obtained by Chen et al. [4] because we used the same DFT code with similar settings. The structural parameters calculated with the hybrid functional are within $1 \%$ of the PBE-GGA results for $\mathrm{C}$ and $\mathrm{Si}$, while the difference
TABLE I. Structural properties of group IV kagome lattices. The interatomic bond lengths in the zigzag chains, $d_{\mathrm{zz}}$, and in the triangles, $d_{\mathrm{tr}}$, are given in $\AA$. The bond angles in the zigzag chain, $\theta_{\mathrm{zz}}$, in the triangles, $\theta_{\mathrm{tr}}$, and between the zigzag chains and the triangles, $\theta_{\mathrm{zt}}$, are given in degrees $\left(^{\circ}\right)$. Values in parentheses are HSE06 results.

\begin{tabular}{lccc}
\hline \hline & CKL & SiKL & GeKL \\
\hline$a$ & $4.457(4.422)$ & $6.941(6.880)$ & $7.442(7.316)$ \\
$c$ & $2.530(2.516)$ & $3.909(3.892)$ & $4.145(4.083)$ \\
$d_{\mathrm{zz}}$ & $1.532(1.517)$ & $2.368(2.349)$ & $2.544(2.498)$ \\
$d_{\mathrm{tr}}$ & $1.499(1.491)$ & $2.333(2.318)$ & $2.479(2.442)$ \\
$\theta_{\mathrm{zz}}$ & $117.7(117.7)$ & $118.2(118.1)$ & $118.4(118.4)$ \\
$\theta_{\mathrm{tr}}$ & $115.1(115.0)$ & $113.8(114.2)$ & $113.5(113.5)$ \\
$\theta_{\mathrm{zt}}$ & $60.0(60.0)$ & $60.0(60.0)$ & $60.0(60.0)$ \\
\hline \hline
\end{tabular}

for the GeKL is about $2 \%$. The bond lengths obtained with the HSE06 functional are always smaller and are believed to be more accurate $[18,19]$. Note that the geometry of the various KLs is rather similar although their size is very different. The SiKL and GeKL are approximately 1.55 and 1.65 times larger than the CKL, respectively, which corresponds roughly to the ratios of the covalent radii of these atoms (1.53 and 1.62), calculated in a diamond structure. All the KLs contain bond angles of $60^{\circ}$ in their structure, which is very small for fourfold coordinated atoms. As shown below, this does not prevent the structures from having large cohesive energies [4].

The stability of carbon kagome lattices was already demonstrated by Chen et al. [4], but it is interesting to see whether the $\mathrm{Si}$ and Ge counterparts are stable too. Therefore, we investigate the dynamical and relative phase stability of these different structures. We start with a study of their structural stability by calculating their phonon spectra. The phonon band structures of CKL, SiKL, and GeKL are given in Fig. 2. The absence of negative (imaginary) phonon frequencies for CKL and SiKL [see Figs. 2(a) and 2(b), respectively] demonstrates the dynamical stability of these structures. Some small imaginary frequencies around the $\Gamma$ point are observed in the phonon spectrum of GeKL. Since these imaginary frequencies belong to an optical mode, this observation indicates an instability of the GeKL structure rather than a computational inaccuracy. The negative phonon mode corresponds to a synchronized rotation of the two triangles in the GeKL unit cell and can be removed by a rotation of these triangles over approximately $12^{\circ}$ in either direction [see Fig. 3(b)]. This distortion leads (a)

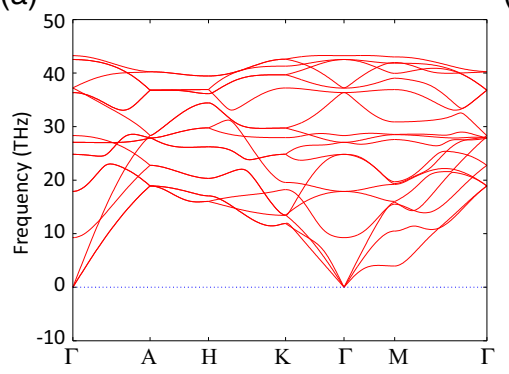

(b)

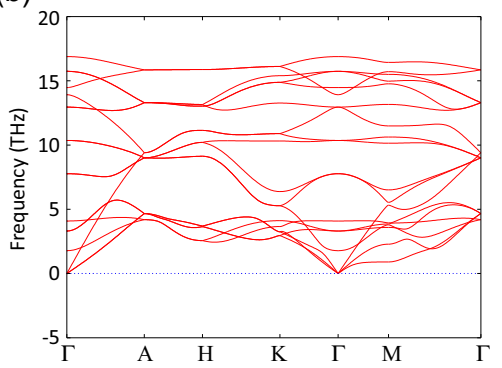

(c)

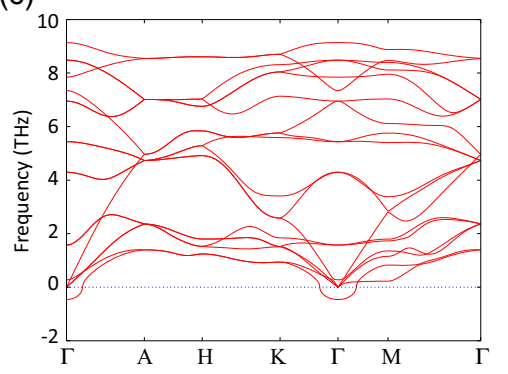

FIG. 2. (Color online) The phonon band spectra of (a) CKL, (b) SiKL, and (c) GeKL as calculated with the GGA functional. The negative frequencies around $\Gamma$ in case of GeKL indicate an instability of the structure. 
(a)

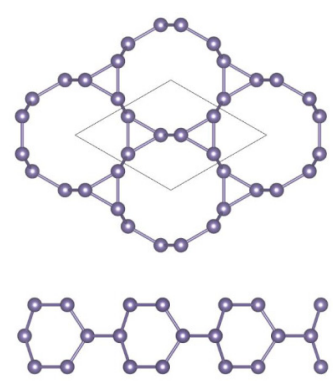

(b)

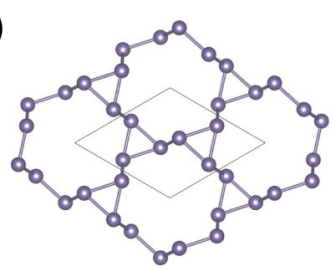

(c)

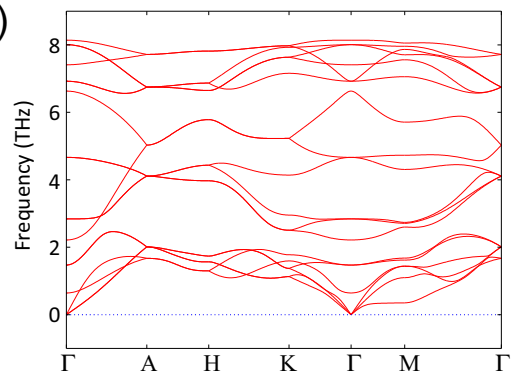

FIG. 3. (Color online) Top (top) and side (bottom) view of the undistorted (a) and (b) GeKL structure. (c) The phonon band spectra of the distorted GeKL.

to an energy gain of only $5 \mathrm{meV} /$ atom and results in a dynamically stable structure, as demonstrated by the absence of negative phonon frequencies in the phonon band spectrum of the distorted structure [Fig. 3(c)]. The smallness of the energy gain makes the distortion unstable at normal temperatures: An $a b$ initio molecular dynamics (AIMD) simulation at $500 \mathrm{~K}$ needs less than $1 \mathrm{ps}$ to switch the direction of the distortion. We also did not observe substantial changes in the electronic properties upon distortion.

To investigate the relative phase stability of the KLs, we calculate their cohesive energies and compare them with those of various related structures. The studied group IV elements all have very stable 3D diamondlike allotropes, but they can also form 2D hexagonal lattice structures called graphene [20,21], silicene, and germanene, respectively [22]. These 2D structures can also be used to make interpenetrated networks (IN), as shown in Figs. 1(b) and 1(d). The interpenetrated graphene network (IGN) is different from the interpenetrated silicene and germanene networks (ISiN and IGeN) in that the latter have a more buckled structure [indicated by the red circle in Fig. 1(d)], just like their 2D counterparts [22]. The IGN was proposed by Chen $e t$ al. as an intermediate structure to create CKL because it is more stable than CKL and can be transformed into the latter by compressing the system [4]. We also compare the KL with the recently proposed layered dumbbell (LD) structure of silicite [1]. The cohesive energies of all the different elements and structures are given in Table II. These cohesive energies are defined as the energy (per atom) needed to separate the atoms of the system. We also show the difference in cohesive energy between the various allotropes and the kagome lattice structure, $\Delta E_{\mathrm{coh}}=E_{\mathrm{coh}}(X)-E_{\mathrm{coh}}(\mathrm{KL})$.

Compared to the diamond structures the kagome lattices of all investigated elements have a similar cohesive energy, i.e., they are about $300 \mathrm{meV}$ less stable. SiKL and GeKL are therefore also realistic candidates to realize 3D kagome lattices.
TABLE II. The cohesive energy $E_{\text {coh }}$ of various $\mathrm{C}, \mathrm{Si}$, and $\mathrm{Ge}$ allotropes. The energies are given in $\mathrm{eV}$.

\begin{tabular}{|c|c|c|c|c|c|c|}
\hline & \multicolumn{2}{|c|}{$\mathrm{C}$} & \multicolumn{2}{|c|}{$\mathrm{Si}$} & \multicolumn{2}{|c|}{$\mathrm{Ge}$} \\
\hline & $E_{\mathrm{coh}}$ & $\Delta E_{\mathrm{coh}}$ & $E_{\mathrm{coh}}$ & $\Delta E_{\mathrm{coh}}$ & $E_{\mathrm{coh}}$ & $\Delta E_{\mathrm{coh}}$ \\
\hline KL & 7.438 & 0.000 & 4.225 & 0.000 & 3.412 & 0.000 \\
\hline diamond & 7.721 & 0.282 & 4.553 & 0.328 & 3.722 & 0.310 \\
\hline $\mathrm{x}$-ene & 7.853 & 0.414 & 3.914 & -0.311 & 3.243 & -0.169 \\
\hline IN & 7.613 & 0.175 & 4.080 & -0.145 & 3.357 & -0.055 \\
\hline LD & 7.212 & -0.226 & 4.370 & 0.145 & 3.552 & 0.140 \\
\hline
\end{tabular}

The fact that the cohesive energy of CKL is $300 \mathrm{meV}$ smaller than diamond could be more or less expected by comparing the cohesive energy of cyclohexane to cyclopropane $\left(\mathrm{C}_{6} \mathrm{H}_{12}\right.$ and $\mathrm{C}_{3} \mathrm{H}_{6}$ ). The $60^{\circ}$ bond angles in the latter induce a strain energy of $368 \mathrm{meV}$ per strained bond angle. This calculated value of the difference in the ring strain between $\mathrm{C}_{6} \mathrm{H}_{12}$ and $\mathrm{C}_{3} \mathrm{H}_{6}$ compares well to the experimental value of $0.39 \mathrm{eV}$ [23] and is of the same order as the difference in cohesive energy between diamond and CKL.

Somewhat surprisingly, the experimentally realized silicene [24] has smaller cohesive energy than the Si kagome lattice by as much as $311 \mathrm{meV}$. This fact means that once created the SiKL is more stable than silicene, but does not imply that it is easier to create the KL. Indeed, it is harder to imagine an easy route towards the creation of SiKL than silicene. A similar observation can be made for Ge. Germanene has a cohesive energy that is $169 \mathrm{meV}$ smaller than that of GeKL. The Si and Ge cases should be contrasted to the C case where graphene has larger cohesive energy than CKL and even diamond. The reason can be found in the preferred fourfold coordination of $\mathrm{Si}$ and $\mathrm{Ge}$ atoms. $s p^{2}$ hybridization is not favorable for these elements as demonstrated by the buckled structure of the 2D hexagonal lattices they form [22]. They favor $s p^{3}$ hybridization and consequently prefer fourfold over threefold coordination. This has also consequences for the stability of the interpenetrated network structures. The INs contain a mixture of threefold and fourfold coordinated atoms, rendering ISiN ( $\mathrm{IGeN}$ ) more stable than silicene (germanene) but less stable than SiKL (GeKL). This excludes the IN structure as a possible intermediate material to create kagome lattices of Si and Ge. Finally, the same trends can be observed for the layered dumbbell structures of $\mathrm{Si}$ and $\mathrm{Ge}$. These materials contain fourfold coordinated atoms but have less distorted bond angles than the KL structures. Therefore, their cohesive energy is in between those of diamond and the KLs. The carbon DL is somewhat exceptional in that it is less stable than the CKL. This can probably be explained by the instability of $\mathrm{C}$ dumbbell configurations [25].

Now that we have demonstrated the stability of the different group IV KLs, we investigate their electronic properties in the next part. The electronic band structure of the different KLs as calculated with the HSE06 functional is shown in Fig. 4. CKL and SiKL show semiconducting behavior with band gaps of 3.427 and $0.616 \mathrm{eV}$, respectively, while GeKL remains gapless. The band gap of CKL $(3.4 \mathrm{eV})$ is considerably smaller than that of diamond (5.3 $\mathrm{eV} \mathrm{[18])} \mathrm{and} \mathrm{graphane}$ (4.4 eV [26]) at the same level of computation (HSE06), but it is still rather large. Similarly, the band gap of SiKL $(0.6 \mathrm{eV})$ 

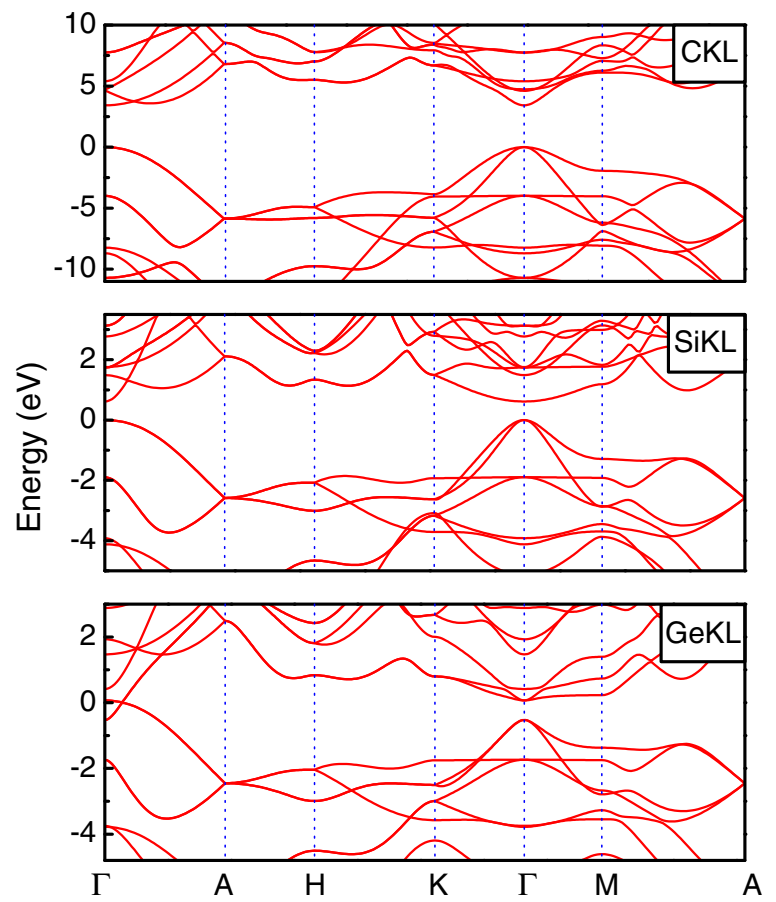

FIG. 4. (Color online) The electronic band structure of CKL, SiKL, and GeKL as calculated with the HSE06 functional. The valence band maximum is put to 0 for CKL and SiKL, while the Fermi level is taken as the origin for GeKL.

is substantially smaller than that of silicon (1.15 eV [18]) and silicane (2.91 eV [27]). We can conclude from these observations that the 3D kagome lattices tend to a have smaller band gap than their diamondlike counterparts. Consequently, it comes as no surprise that $\mathrm{GeK}$ has overlapping bands because the bulk germanium has a band gap of only $0.8 \mathrm{eV}$ [18].

We examine the electronic properties of CKL and SiKL first and postpone the discussion of GeKL to the last part of this work. Except for the quantitative difference in the band gap, the electronic band structure of CKL and SiKL look rather similar at first sight. However, further inspection shows that they are qualitatively different. In Fig. 5, the orbital projected density of states and band structure as calculated within the GGA are shown. We make a distinction between $s, p_{x / y}$, and $p_{z}$ orbital character. Both CKL and SiKL are found to be direct-gap materials, which should be contrasted to the other $\mathrm{C}$ and $\mathrm{Si}$ allotropes that show indirect gaps. The valence band states show strong $p_{x / y}$ character for both CKL and SiKL, but the conduction bands have different symmetry. In the case of CKL, the conduction bands have also $p_{x / y}$ character, making this material into a true direct-gap material [4]. The conduction bands of SiKL, on the other hand, have mixed symmetry (predominantly $s$ like). This qualitative difference between the band structures of SiKL and CKL is explicitly demonstrated by the imaginary part of the dielectric function, depicted in Fig. 5(c). The dipole-allowed transitions in CKL start from the first conduction band, but no such transitions are observed for SiKL. For SiKL the optical transitions only start from the fifth conduction band (including degeneracies), which has the same symmetry as the first conduction band of CKL (i.e., $p_{x / y}$ ). (a)

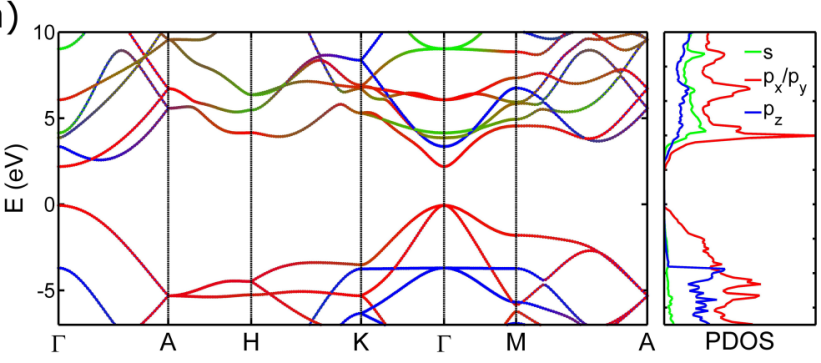

(b)

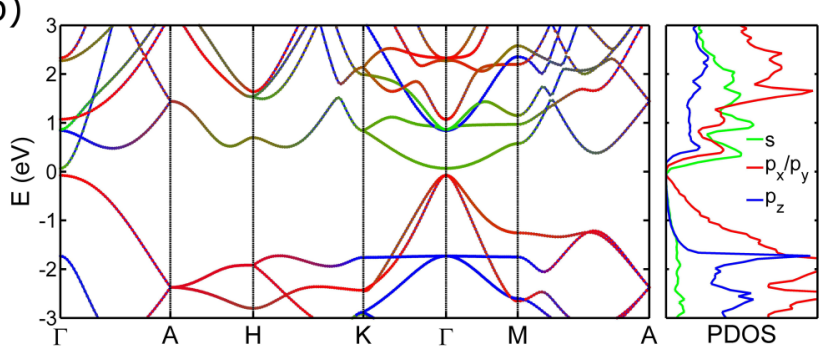

(c)
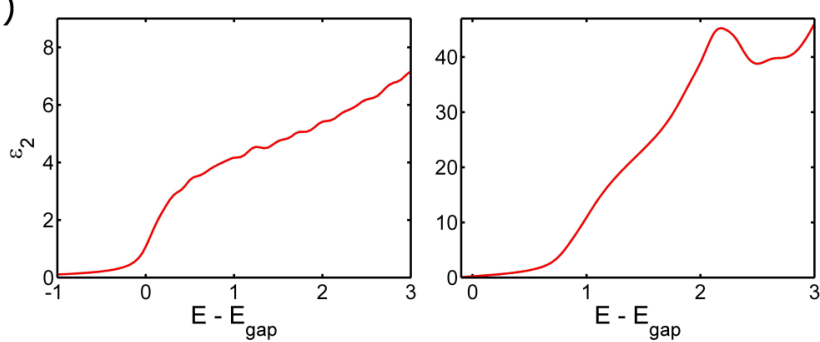

FIG. 5. (Color online) The orbital projected electronic band structure and density of states of CKL (a) and SiKL (b) calculated with the PBE-GGA $x c$ functional. The energy corresponding to the valence band maximum is put to zero. (c) The imaginary part of the dielectric function, $\epsilon_{2}$ of CKL (left) and SiKL (right).

The effective masses of the various possible charge carriers in CKL and SiKL are given in Table III. Note that there is a large difference between the GGA-PBE and HSE06 results for the effective masses in SiKL. This is related with the large quantitative difference in the electronic band gap, 0.144 vs $0.616 \mathrm{eV}$, resulting from the two $x c$ functionals. The HSE06 results are obviously more reliable [28,29]. We can make a distinction between the direction along the zigzag chains in the kagome lattice (perpendicular) and the plane normal to these chains (parallel). In CKL, the electrons and holes have

TABLE III. Electronic properties of $\mathrm{C}$ and Si kagome lattices. The band gap is given in $\mathrm{eV}$ and the effective masses are given in unit of the free electron mass.

\begin{tabular}{lrrrrr}
\hline \hline & \multicolumn{2}{c}{ CKL } & & \multicolumn{2}{c}{ SiKL } \\
\cline { 2 - 3 } \cline { 5 - 6 } & GGA-PBE & HSE06 & & GGA-PBE & HSE06 \\
\hline$E_{\text {gap }}$ & 2.257 & 3.427 & & 0.144 & 0.616 \\
$m_{\mathrm{e}}^{\|}$ & 0.141 & 0.156 & & 1.338 & 2.523 \\
$m_{\mathrm{lh}}^{\|}$ & -0.119 & -0.127 & & -0.072 & -0.182 \\
$m_{\mathrm{hh}}^{\|}$ & -0.511 & -0.469 & & -0.132 & -0.351 \\
$m_{\mathrm{e}}^{\perp}$ & 1.229 & 1.063 & & 0.116 & 0.230 \\
$m_{\mathrm{h}}^{\perp}$ & -0.913 & -0.819 & & -1.023 & -1.889 \\
\hline \hline
\end{tabular}


light masses in the parallel direction while their masses are free-electron-like in the direction along the zigzag chain. The same is true for the holes in SiKL, but the electrons show opposite behavior in that case. This can be explained by the different orbital symmetries of the respective bands. The hole states consist of $p_{x / y}$ orbitals which give rise to large dispersion (small effective mass) in the $x y$ plane and little dispersion (large effective mass) in the direction perpendicular to this plane because of the size of the orbital overlap. The same is true for the electron states in CKL which also have $p_{x / y}$ symmetry. The electron states of SiKL have mixed orbital character with no obvious preference in direction (mostly $s$ like and similar contributions of $p_{x / y}$ and $p_{z}$ ). However the bonds along the zigzag chain are significantly shorter than the in-plane bonds (see Table I) and lead consequently to lower effective masses.

In the last part of this work, we treat the electronic properties of GeKL to demonstrate the role of orbital frustration on the electronic properties. We showed before that GeKL shows semimetallic behavior. In their recent study, Chen et al. showed how the gapped state in CKL originates from the orbital frustration that is built in into the kagome lattice structure [4]. This physical origin suggests that an increase in the orbital frustration might induce an electronic gap in GeKL. This can be achieved by increasing the orbital interaction by compressing the GeKL crystal. In Fig. 6 we show the evolution of the electronic band structure, calculated with the HSE06 functional, when going from 0 to $6 \%$ uniform compression, in which $1 \%$ compression corresponds to a reduction of the lattice parameters with $1 \%$. Since we are only interested in the electronic properties, we neglect the distortions in the GeKL structure. It can be seen that a gap appears at a compression of $4 \%$. Although this large compression is probably not very practical to achieve in experiment, the opening of the band

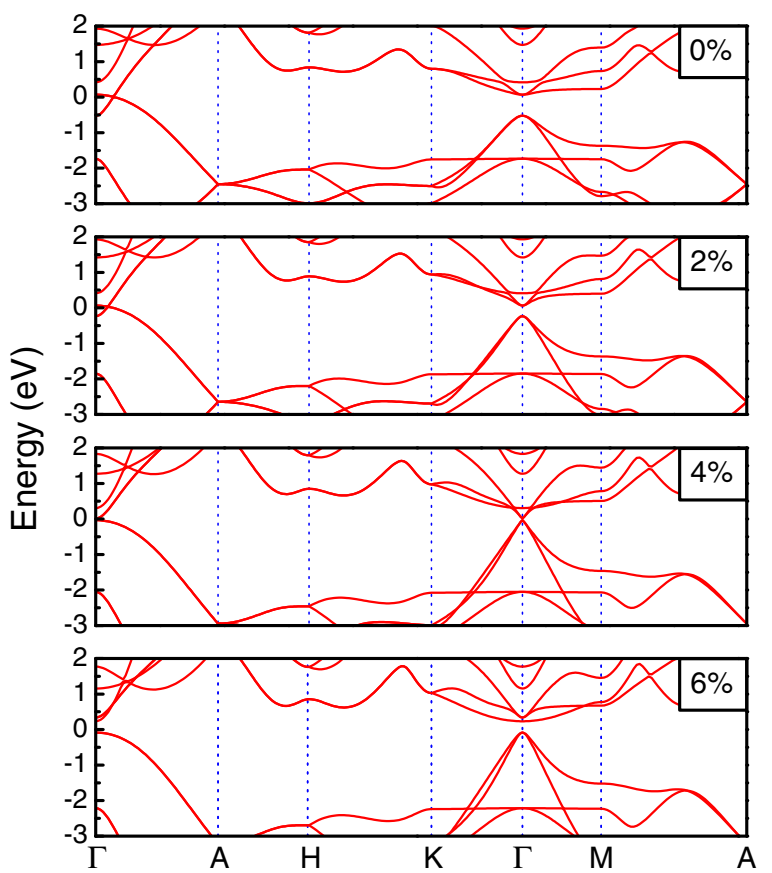

FIG. 6. (Color online) The band structure of GeKL under various compressions calculated with the HSE06 functional.
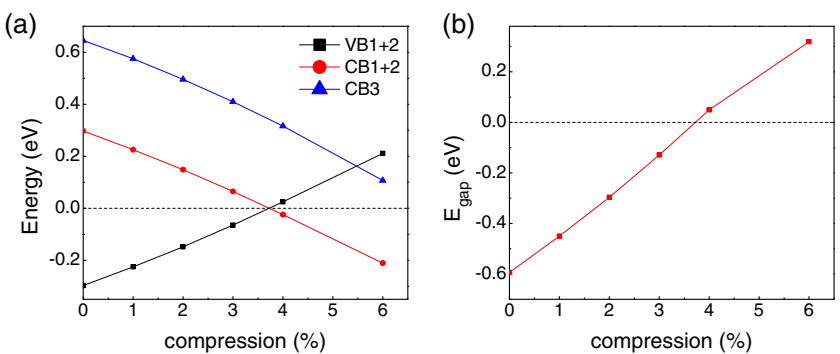

FIG. 7. (Color online) The positions of the valence and conduction bands relative to the middle of the band gap (a) and the electronic band gap (b) as a function of compression.

gap demonstrates the importance of orbital frustration in the process.

In Fig. 7, we show the position of the relative positions of the valence band maxima (VBM) and conduction conduction band minima (CBM), together with the size of the electronic band gap as a function of compression. The band gap is taken negative in the semimetallic case and it can be seen to be a continuous function of the compression [see Fig. 7(b)]. Note that there is a kink at higher compressions, which is due to a change of bands that contribute to the band gap. As can be seen in Fig. 7(a), this change occurs at approximately 5.5\%. Because the behavior of the two bands (CB1+2 and CB3) under compression is similar, the band gap will converge to a value of $0.3 \mathrm{eV}$ for compressions larger than $5.5 \%$.

\section{SUMMARY AND CONCLUSIONS}

We performed an in-depth $a b$ initio study of the structural and electronic properties of 3D kagome lattices of group IV elements. We found that such kagome lattices are stable for C, $\mathrm{Si}$, and $\mathrm{Ge}$, and have cohesive energies that are about $30 \mathrm{meV}$ lower than the corresponding diamond crystals. Heavier group IV elements give rise to metallic behavior and we did not investigate them any further. The structural parameters and electronic properties were calculated with both GGA and hybrid functionals. The kagome lattices made from carbon and silicon were found to be semiconductors with band gaps of 3.4 and $0.6 \mathrm{eV}$, respectively. We investigated the symmetries of the valence and conduction band states and found them to be very different for CKL and SiKL. Although both materials are direct band gap semiconductors, CKL allows for direct dipole transitions between the VBM and CBM while this is not the case for SiKL. The reason for this is the different symmetries of the lower conduction bands.

Germanium kagome lattices were shown to be dynamically unstable to synchronized rotations of the two triangles in the unit cell over $12^{\circ}$. They exhibit semimetallic behavior but an electronic band gap can be opened by an increase of the orbital frustration interaction by compression. The gap opens at a compression of $4 \%$ and converges to a value of $0.3 \mathrm{eV}$.

\section{ACKNOWLEDGMENTS}

This work was supported by the Fonds Wetenschappelijk Onderzoek (FWO-Vl). The computational resources and services used in this work were provided by the VSC (Flemish Supercomputer Center), funded by the Hercules Foundation and the Flemish Government - department EWI. 
[1] S. Cahangirov, V. O. Özçelik, A. Rubio, and S. Ciraci, Phys. Rev. B 90, 085426 (2014).

[2] P. De Padova, P. Vogt, A. Resta, J. Avila, I. Razado-Colambo, C. Quaresima, C. Ottaviani, B. Olivieri, T. Bruhn, T. Hirahara, T. Shirai, S. Hasegawa, M. C. Asensio, and G. Le Lay, Appl. Phys. Lett. 102, 163106 (2013).

[3] S. Cahangirov, V. O. Özçelik, L. Xian, J. Avila, S. Cho, M. C. Asensio, S. Ciraci, and A. Rubio, Phys. Rev. B 90, 035448 (2014).

[4] Y. Chen, Y. Y. Sun, H. Wang, D. West, Y. Xie, J. Zhong, V. Meunier, M. L. Cohen, and S. B. Zhang, Phys. Rev. Lett. 113, 085501 (2014).

[5] C. Ruegg, Nat. Phys. 6, 837 (2010).

[6] K. Matan, T. Ono, Y. Fukumoto, T. J. Sato, J. Yamaura, M. Yano, K. Morita, and H. Tanaka, Nat. Phys. 6, 865 (2010).

[7] G.-B. Jo, J. Guzman, C. K. Thomas, P. Hosur, A. Vishwanath, and D. M. Stamper-Kurn, Phys. Rev. Lett. 108, 045305 (2012).

[8] C. Nisoli, R. Moessner, and P. Schiffer, Rev. Mod. Phys. 85, 1473 (2013).

[9] L. Balents, Nature (London) 464, 199 (2010).

[10] G. Kresse and J. C. Furthmüller, Comput. Mater. Sci. 6, 15 (1996).

[11] G. Kresse and J. C. Furthmüller, Phys. Rev. B 54, 11169 (1996).

[12] J. P. Perdew, K. Burke, and M. Ernzerhof, Phys. Rev. Lett. 77, 3865 (1996).

[13] S. Heyd, G. E. Scuseria, and M. Ernzerhof, J. Chem. Phys. 118, 82078215 (2003).

[14] G. Kresse and D. Joubert, Phys. Rev. B 59, 1758 (1999).
[15] B. Adolph, J. Furthmüller, and F. Bechstedt, Phys. Rev. B 63, 125108 (2001).

[16] P. E. Blöchl, Phys. Rev. B 50, 17953 (1994).

[17] H. J. Monkhorst and J. D. Pack, Phys. Rev. B 13, 5188 (1976).

[18] K. Hummer, J. Harl, and G. Kresse, Phys. Rev. B 80, 115205 (2009).

[19] M. Schlipf, M. Betzinger, C. Friedrich, M. Ležaić, and S. Blügel, Phys. Rev. B 84, 125142 (2011).

[20] K. S. Novoselov, A. K. Geim, S. V. Morozov, D. Jiang, Y. Zhang, S. V. Dubonos, I. V. Grigorieva, and A. A. Firsov, Science 306, 666 (2004).

[21] K. S. Novoselov, D. Jiang, F. Shedin, T. J. Booth, V. V. Khotkevich, S. V. Morozov, and A. K. Geim, Proc. Natl. Acad. Sci. USA 102, 10451 (2005).

[22] S. Cahangirov, M. Topsakal, E. Aktürk, H. Sahin, and S. Ciraci, Phys. Rev. Lett. 102, 236804 (2009).

[23] N. Cohen and S. W. Benson, Chem. Rev. 93, 2419 (1993).

[24] P. Vogt, P. De Padova, C. Quaresima, J. Avila, E. Frantzeskakis, M. C. Asensio, A. Resta, B. Ealet, and G. Le Lay, Phys. Rev. Lett. 108, 155501 (2012).

[25] V. Ongun Özçelik, D. Kecik, E. Durgun, and S. Ciraci, J. Phys. Chem. C 119, 845 (2015)

[26] M. N. Amini, O. Leenaerts, B. Partoens, and D. Lamoen, J. Phys. Chem. C 117, 16242 (2013).

[27] V. Zólyomi, J. R. Wallbank, and V. I. Fal'ko, 2D Materials 1, 011005 (2014).

[28] F. Tran and P. Blaha, Phys. Rev. Lett. 102, 226401 (2009).

[29] J. P. Perdew, A. Ruzsinszky, J. Tao, V. N. Staroverov, G. E. Scuseria, and G. I. Csonka, J. Chem. Phys. 123, 062201 (2005). 